\title{
Meningeal Leukemia
}

National Cancer Institute

\section{Source}

National Cancer Institute. Meningeal Leukemia. NCI Thesaurus. Code C94754.

Infiltration of the meninges by an acute or chronic leukemia. 